\title{
AN AUDIT OF PATIENTS REFERRED FOR NERVE CONDUCTION STUDY IN A TERTIARY CARE HOSPITAL IN BANGLADESH
}

\author{
CHOWDHURY RN ${ }^{1}$, HUSSAIN ME ${ }^{2}$, ISLAM MN ${ }^{3}$, HOSEN ${ }^{4}$, KHAN AFMA M ${ }^{5}$, MD FERDOUS MIAN 6
}

\begin{abstract}
Background: To examine the pattern and burden of neurologic disorders at electrophysiology lab of a tertiary care centre.

Methodology: This retrospective chart review was carried out from the records and notes of electrophysiology lab in National Institute of Neurosciences and Hospital (NINS) from January to December 2013. A total of 1372 patients were evaluated with nerve conduction study (NCS) and electromyography (EMG) during this period.
\end{abstract}

Result: Majority of the patients (67.6\%) presented after forty with a mean age at presentation of $48.11 \pm 17.3$ years. The male patients (55.2\%) predominated. Carpal tunnel syndrome (CTS) was the most common condition (19.2\%) observed, followed by different form of polyneuropathy namely Guillain Barre Syndrome (GBS) (6.04\% with $50 \%$ being Acute inflammatory demyelinating polyneuropathy (AIDP), chronic inflammatory demyelinating polyneuropathy (CIDP) (3.27\%), sensory motor polyneuropathy 3.13\% and multifocal acquired motor axonopathy (MAMA) $2.55 \%$. Though plexopathy and radiculopathy were rare (1.09 and $0.94 \%$ respectively), anterior horn cell disease was not that uncommon (8.73\%). Disorders of muscle and neuromuscular junction (myasthenia gravis) were seen in $5.1 \%$ and $1.89 \%$ patient. Other various conditions (e.g. stroke, cerebral palsy, myelopathy) were observed in 10.05\%. NCS and EMG were found to be normal in 270 patients (19.6\%).

Conclusion: Wide ranges of neurological problems are often referred to electrophysiology lab. Where ever the facilities and expert hands are available, these tests can help in diagnosing and classifying these cases.

Key words: Nerve conduction study (NCS), electromyography (EMG), electrophysiology, neurophysiology.

\section{Background:}

The overall global burden of neurologic disease is approximately $20 \%$, the majority being shared by the developing countries ${ }^{1}$. The incidence of neurologic disorder in UK is $0.6 \%$ with an overall $6 \%$ lifetime prevalence rate $^{2}$. Experienced clinicians will make around 90\%
J Dhaka Med Coll. 2014; 23(1) : 102-108.

of neurological diagnoses on history alone, with a lesser contribution from examination and investigation. Investigation may include assessment of structure (imaging) and function (neurophysiology) ${ }^{3}$. Neurophysiology may be regarded as the clinical examination with the ability to "probe" nerves and muscles in a

1. Dr Rajib Nayan Chowdhury, Assistant Professor (Neurophysiology), National Institute of Neurosciences and Hospital, Dhaka

2. Dr. Md Enayet Hussain, Assistant Professor of Neurology, National Institute of Neurosciences and Hospital, Dhaka

3. Dr Md Nahidul Islam, Registrar, Neurology, National Institute of Neurosciences and Hospital, Dhaka

4. Dr Mostafa Hosen, Associate Professor of Neurology, Sylhet MAG Osmani Medical College Hospital

5. Dr A.F.M Al Masum Khan, Medical Officer (Neurophysiology), National Institute of Neurosciences and Hospital, Dhaka

6. Dr Md Ferdous Mian, Medical Officer (Clinical Neurology), National Institute of Neurosciences and Hospital, Dhaka

7. Dr A T M Hasibul Hasan, Registrar, Neurology, Dhaka Medical College Hospital

8. Dr Md Badrul Alam Mondal, Professor of Neurology and Joint Director, National Institute of Neurosciences and Hospital, Dhaka

9. Professor Quazi Deen Mohammad, Professor of Neurology and Joint Director, National Institute of Neurosciences and Hospital, Dhaka

Correspondence : Dr ATM Hasibul Hasan, Registrar, Neurology, Dhaka Medical College Hospital . Email: parag007us@gmail.com . Phone-01763498663.

\section{Table not found}


different manner. Electrodiagnostic procedures in neurophysiology lab include nerve conduction studies (NCS) and/or needle electromyography (nEMG), which have historically been performed by physicians with neurology or physical and rehabilitative medicine (PM\&R) training.

Nerve conduction studies (NCS) involve placing electrodes on the skin overlying peripheral nerves and recording compound action potentials (the sum of all the individual nerves' action potentials) following nerve stimulation as the impulse travels down the nerve. Electromyography (EMG) involves recording compound motor action potentials (CMAPs) over muscles in response to motor nerve stimulation. Fine concentric needle electrodes are inserted into muscle belly and the potentials from individual motor units recorded $^{3}$. The electrodiagnostic tests are often considered as an extension of clinical examination in diagnosing the neurological disorders. At earlier times every textbook or article on NCS/EMG emphasized that these tests do not replace a careful history and examination of the patient and are an extension of the clinical assessment ${ }^{4}$.

Neurology was introduced as a specialty in Bangladesh during the 1960s'. Apart from infection and malnutrition, an excess burden of cerebrovascular disease and stroke at an early age is related to a high risk of mortality and morbidity in Bangladesh ${ }^{5,6}$. Due to lack of facilities elsewhere in the country varieties of neurological problems are referred to National Institute of Neurosciences and Hospital (NINS), inaugurated in September 2012. Previously we did not have any published data regarding the pattern of disease seen in electrophysiology room. We therefore tried to audit the nature of neurologic involvement among patients referred to the largest electrophysiology lab in Bangladesh.

\section{Methods:}

This is a retrospective chart review. We reviewed the records and notes of electrophysiology lab in National Institute of
Neurosciences and Hospital (NINS) from January to December 2013. A total of 1372 patients were evaluated with nerve conduction study during this period.

NINS, the centre of excellence and one of the highest centers of referral for any neurological disorder has 300 inpatient beds. The electrophysiology lab has 4 machines for NCS and EMG and several consultant neurologists who are trained in electrophysiology both at home and abroad, with fellowship in the respective field. All patient referred to electrophysiology lab were eligible for inclusion except those diabetes, chronic kidney disease or receiving chemotherapy. Each of the referred patients was examined by the Consultant Neurologist who also performed the NCS and EMG. Patient's problem was initially diagnosed clinically with proper history, examination and with help of investigations in required cases at inpatient or outpatient department. Later they were advised for NCS and EMG to detect the distribution and type of abnormalities of individual patients.

Nerve conduction parameters were derived from computer based cursor assignments of supramaximally stimulated response waveforms and were normalized to standardized temperatures using linear correction factors. The parameters evaluated included the distal motor latency (DML), compound muscle action potential (CMAP) amplitude measured from baseline to the negative peak, mean $\mathrm{F}$-wave latency ( $\mathrm{F}$-wave), distal sensory latency (DSL) measured to the negative peak, and sensory nerve action potential (SNAP) amplitude measured from the negative to positive peak. Measurements were flagged as abnormal if they were outside normal limits after adjusting for patient's age and height. Normal limits were set at the 97.5th percentile for latency parameters and at the 2.5th percentile for amplitude relative to disease free controls. Percentiles between 90 and 97.5 for latency and between 2.5 and 10 for amplitude were flagged as borderline. All nerve conduction parameters could not be 
obtained in every encounter because of technical errors or patient-specific factors, including severe artifact and A-waves obscuring the onset of $\mathrm{F}$ waves. The mean $\mathrm{F}$ wave latency was calculated when at least three $\mathrm{F}$-wave responses were measured. The mean F-wave latency was flagged as absent if sufficient $F$ wave responses were not available. The abnormality rate for a parameter was defined as the percentage of nerves outside normal limits. Absent responses were considered abnormal for distal motor latency (DML), compound motor action potential (CMAP), distal sensory latency (DSL), and sensory nerve action potential (SNAP).

Information regarding the demographic and clinical profile was gathered through a questionnaire. Diseases were categorized under 7 broad headings, namely, peripheral nerve entrapment, polyneuropathy, plexopathy, radiculopathy, anterior horn cell disease, neuromuscular junction disorder and disorder of muscles, depending on location of abnormalities. Analysis was done using SPSS version 16.0.

\section{Results:}

Majority of the patients (67.6\%) presented after the age of forty years. Highest number of patient (349) was seen in 41-50 years age group. The mean age at presentation was $48.11 \pm 17.3$ years. The male patients $(55.2 \%)$ predominated (Table-I).

Carpal tunnel syndrome (CTS) was the most common condition (19.2\%) observed at the lab. Other peripheral nerve entrapment in the form of mononeuropathy was present in 81 (5.9\%) patients, out of which peroneal nerve entrapment was commonest (30.1\%). Different form of polyneuropathy namely Guillain Barre Syndrome (GBS), chronic inflammatory demyelinating polyneuropathy (CIDP), sensory motor polyneuropathy, traumatic neuropathy, multifocal acquired motor axonopathy (MAMA), hereditary neuropathy and sensory neuropathy in $6.04 \% 3.27 \%, 3.13 \%, 2.55 \%, 1.53 \%$ and $1.45 \%$ respectively. Mononeuritis multiplex and multifocal motor neuropathy with conduction block (MMNCB) was relatively rare (9 and 5 patients respectively). Acute inflammatory demyelinating polyneuropathy (AIDP) was commonest (49.3\%) form of GBS, followed by acute motor axonal neuropathy (AMAN) in 30.1\% (Table-II).

\section{Table-I}

Socio demographic profile of the patients $(n=1372)$

\begin{tabular}{llcc}
\hline Parameter & $\mathrm{n}$ & $\%$ \\
\hline Age & $<10$ yrs & 24 & 1.7 \\
& $11-20$ yrs & 91 & 6.7 \\
& $21-30 \mathrm{yrs}$ & 14 & 10.7 \\
& 31-40yrs & 182 & 13.3 \\
& 41-50 yrs & 349 & 25.5 \\
& $51-60$ yrs & 204 & 14.8 \\
& $61-70$ yrs & 197 & 14.3 \\
& $>70$ yrs & 178 & 13 \\
Sex $\quad$ Male & 757 & 55.2 \\
& Female & 615 & 44.8 \\
\hline
\end{tabular}

Though plexopathy and radiculopathy were relatively rare (1.09 and $0.94 \%$ respectively), anterior horn cell disease was not that uncommon. In the later group, motor neuron disease was diagnosed in $3.13 \%$, monomelic amyotrophy (MMA) in $1.38 \%$ and other disorders involving anterior horn cell in $3.71 \%$ patients. Disorders of muscle and neuromuscular junction (myasthenia gravis) were seen in $5.1 \%$ and $1.89 \%$ patient. Among the patients with disorders of muscle, non-inflammatory myopathy was most frequent diagnosis $(52.9 \%)$, followed by myotonic dystrophy (30\%) and inflammatory myopathy (17.1\%). Other various conditions (e.g. stroke, cerebral palsy, myelopathy) were observed in $10.05 \%$. NCS and EMG were found to be normal in 270 patients (19.6\%). 
Table-II

Findings at electrophysiology lab $(n=1372)$

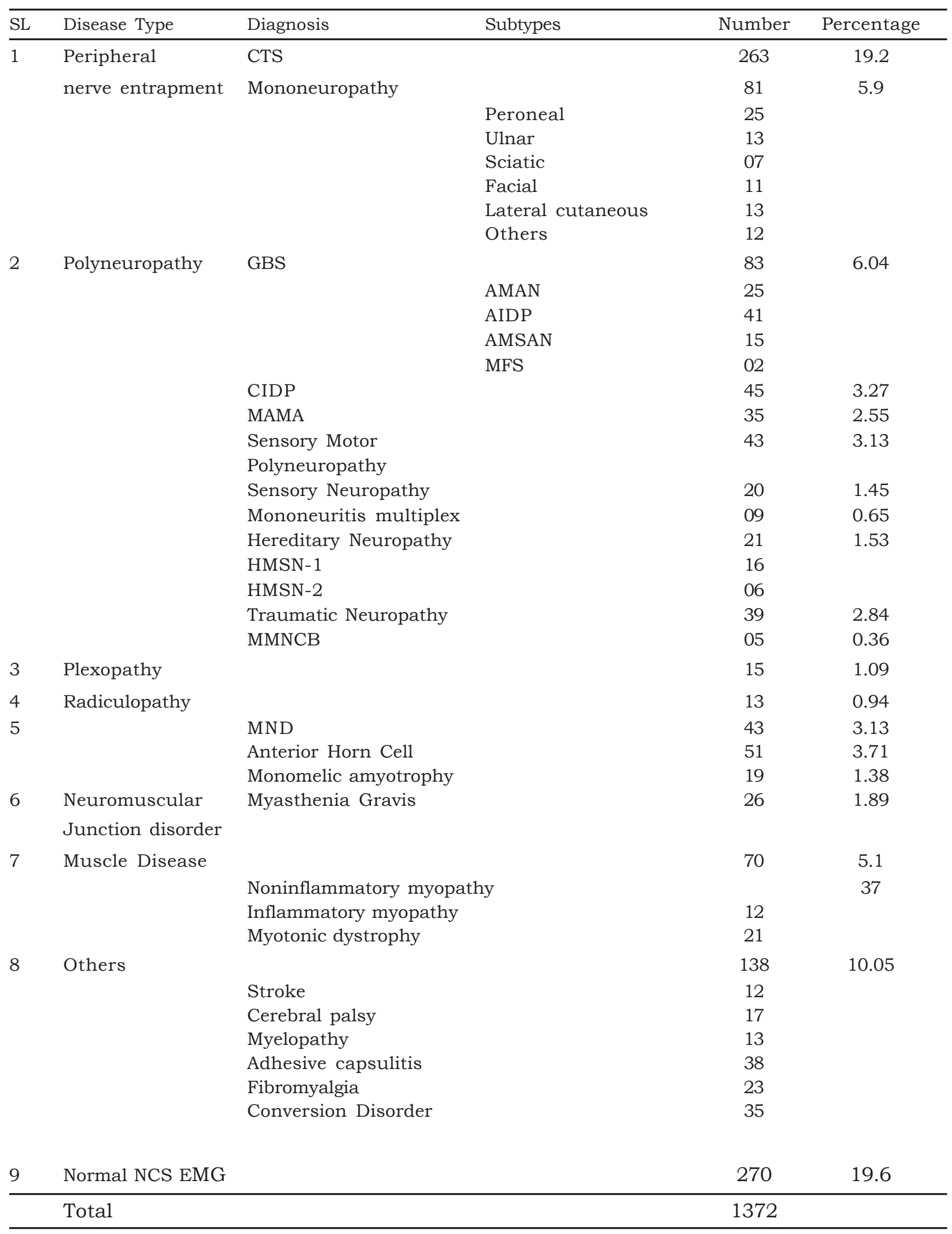




\section{Discussion:}

Nerve conduction studies as part of the peripheral neurological examination are an extension of the clinical history and examination and are important in the management of cranial and peripheral neuromuscular disease as well as contributing to diagnosis of spinal cord lesions. NCS can be extremely useful both in localizing the lesions and determining the pathological processes responsible. We conducted this study to get a glimpse disease burden and pattern of various form of neuropathy and myopathy in neurology department of NINS.

The age and male sex related increase of neurological disorders are a common trend that is also seen in studies conducted in Dhaka Medical College Hospital (DMCH) ${ }^{7,8}$. Different forms of peripheral neuropathy, MND or myopathy were relatively less frequently $(2.8$, 0.5 and $0.3 \%$ respectively) seen at neurology out patient in $\mathrm{DMCH}^{8}$. All these disorders require electrophysiologic evaluation.

MacDonald et al reported ${ }^{9}$ that the age and sex adjusted incidence (per 100,000/ year) and life time prevalence (per 1000 population) of different polyneuropathy (excluding diabetics and alcoholics), compressive mononeuropathy (except CTS) in the community were 15, 49 and 1, 2 respectively. Neurophysiological studies performed in best hands, gives enormous facts in the diagnosis or elimination of PN existence. EMG, NCS may act as the sheet anchor of diagnosis ${ }^{10,11,12}$.

In our series among the compressive neuropathy, CTS was quite a common finding in nerve conduction. Carpal tunnel syndrome (CTS) is the most common focal neuropathy and a major cause of disability in the United States ${ }^{13}$. There was considerable controversy regarding the need for electrophysiology in carpal tunnel syndrome. Electrophysiology has been proposed as the standard of care for diagnosing carpal tunnel syndrome with a recommendation that it should be performed before surgery in all cases. ${ }^{14}$ This view has been supported by recent articles in the JAMA ${ }^{15}$ and the $\mathrm{BMJ}^{16}$. So, the utility of NCS in such disorder, which includes objective confirmation of nerve involvement, grading of severity, is well accepted worldwide ${ }^{17}$.
Peroneal neuropathy is one of the most common focal neuropathy in lower limb ${ }^{18}$, while ulner neuropathy is the second most frequent neuropathy in upper extremity ${ }^{19}$. Peroneal neuropathy was more frequent in this study. Electrodiagnosis can play a pivotal role in the evaluation of these neuropathies. It can document the presence of a mononeuropathy; localize the lesion to any of several locations in the wrist, forearm, or elbow; and distinguish a mononeuropathy from a plexopathy, radiculopathy, polyneuropathy, or motor neuron disease. In addition to establishing a diagnosis and locating a level of lesion, EDS can also give prognostic informations ${ }^{20,21}$.

Next to compressive neuropathy, we found a considerable number in inflammatory neuropathy (GBS, CIDP). The crude incidence rate of GBS in Bangladesh ranges from 1.5-1.7/ $100,000 /$ year $^{22}$. In contrary Islam $Z$ et $\mathrm{al}^{23}$, who reported a high frequency (56\%) of AMAN at electrophysiology, we observed AIDP in 49\%. This is probably due to the fact that they conducted the study in patients aged less than 15 years, whereas we had patients from all age groups. Electro-diagnostic studies are helpful in determining the diagnosis, differentiating and classifying variety of GBS and also their response to treatment and prognosis ${ }^{23}$. But it is generally thought that test results obtained early in the course of illness may lead to misclassification of the subtype and that serial nerve-conduction studies are therefore important for accurate subtype classification. ${ }^{25}$ On the other hand multifocal demyelination is a diagnostic hallmark of CIDP ${ }^{26}$. The distal nerve terminals may be preferentially affected and after successful treatment, patients show an obvious increase in the amplitude of distally evoked compound muscle action potential, suggesting resolution of distal conduction block ${ }^{27}$. Though the number of patients with hereditary neuropathy was very low, the disorder of anterior horn cell was not uncommon. Electrophysiology allows identification of LMN features of MND in both clinically affected and as yet clinically silent regions and thus helps to make an earlier working diagnosis. Typical electromyographic features of MND include evidence of active 
denervation (positive sharp waves, fibrillation potentials, fasciculation potentials) and chronic denervation evidenced by large motor unit potentials $^{28}$.

Neurophysiologic methods are of great importance in order to recognize myasthenic patients among those with muscle fatiguability and to follow the effect of different therapeutic measures ${ }^{29}$. Thus, electromyography can be used to investigate the neuromuscular junction. Repetitive stimulation of a nerve with trains of electrical impulses at 3-15/s does not normally result in a significant fall-off in the amplitude of the resulting muscle action potential. However, such a decrement seen in myasthenia gravis provides one of the key diagnostic features ${ }^{3}$. Muscle disorders were not too infrequent in our study. At neurology out patient department of $\mathrm{DMCH}$, it was reported to be $0.3 \%$ of all neurologic disorders ${ }^{8}$. Abnormalities in the shape and size of muscle potentials can also help in the differential diagnosis of denervation and structural muscle diseases. Myopathies caused by metabolic abnormalities (causing electromechanical dissociation rather than loss of fiber structure) show no changes on needle $\mathrm{EMG}^{3}$. Such test also record abnormal spontaneous activity arising from muscles at rest, such as fibrillations or myotonic discharges and helps in differentiating different myopathies.

We had some limitations in this study. A potential limitation of electrodiagnostic studies is that they are able to test only the large, myelinated nerve fibers. This limits their sensitivity in detecting neuropathies of the small nerve fibers (i.e., those with pain, temperature, and autonomic functions). In these cases, a specialized test directed at autonomic functions, and other nonelectrodiagnostic tests (e.g., epidermal skin biopsy) may yield the diagnosis. Moreover, this study does not give any idea about the distribution of neurologic disorder in Bangladesh.

\section{Conclusion:}

Appropriate clinical examination determines the site of the lesion by assessing the distribution of weakness, reflex changes, and sensory loss, whereas neurophysiology examine not only the distribution but also the type of abnormalities as detected in the nerve conduction studies and EMG. Wide range of neurologic disorders ranging from, peripheral neuropathy to muscle diseases are often referred by physicians to electrophysiology lab. For the neurologist or other referring doctor, it is equally vital that the clinical questions asked are explicit and answerable for the most to be gained from electrophysiology, which is a considerable investment in time and skills for the investigator and tolerance of discomfort in the patient.

Conflict of interest: None.

Funding: None.

\section{Authors Contribution:}

RN C was involved concept, data collection and revision of manuscript for this study. ATM HH was involved in data analysis and writing the article. The rest were involved in design, data collection and analysis. All the authors have read and approved the final version of the manuscript.

\section{References:}

1. Burton KJ and Allen S. A review of neurological disorders presenting at a paediatric neurology clinic and response to anticonvulsant. Ann Trop Pediatric 2003; 23:139 - 143.

2. MacDonald BK, Cockerell OC, Sander JWAS, Shorvon SD. The Incidence and Lifetime Prevalence of Neurologic Disorder in a prospective community-based study in the UK. Brain 2000; 123:665-76.

3. Allen CMC, Lueck CJ, Dennis M. Neurologic disease. In: Colledge NR, Walker BR, Ralston SH, editors. Davidson's Principles and Practice of Medicine. $21^{\text {st }}$ edition. Elsevier Limited; 2010: pp1142 .

4. G Fuller. How to get the most out of nerve conduction studies and electromyography. J Neurol Neurosurg Psychiatry 2005;76(Suppl II):ii4 1-ii46.

5. MacKay J, Mensah GA. The Atlas of Heart Disease and Stroke. Geneva: WHO Press; 2004

6. Bhopal R, Rahemtulla T, Sheikh A. Persistent high stroke mortality in Bangladeshi populations. BMJ 2005;331: 1096-1097.

7. Rajib Nayan Chowdhury, ATM Hasibul Hasan, Yusuf Ur Rahman et al. Pattern of neurological 
disease seen among patients admitted in tertiary care hospital. BMC Research Notes 2014, 7:202.

8. RN Chowdhury, ATM H hasan, KM Rahman et al. Spectrum of Neurological Disorders: Experience in Specialized Outpatient Clinic in Bangladesh. J MEDICINE 2012; 13 : 39-42.

9. MacDonald BK, Cockerell OC, Sander JWAS, Shorvon SD. The Incidence and Lifetime Prevalence of Neurologic Disorder in a prospective community-based study in the UK. Brain 2000; 123:665-76.

10. Donofrio PD Albers JW. AAEM minimonograph 34: polyneuropathy : classification by nerve conduction studies and electromyography. Muscle Nerve 1990; 13: 889 - 903.

11. Mc Leod JG, Investigation of peripheral neuropathy. J. Neurol Neurosurg psychiatry 1995 ; 58: 274-83.

12. Thrush D. Investigation of peripheral neuropathy. Br J. Hosp Med 1992; 48: 13- 22.

13. Blanc PD, Faucett J, Kennedy JJ, Cisternas M, Yelin E. Self-reported carpal tunnel syndrome: predictors of work disability from the National Health Interview Survey Occupational Health Supplement. Am J Ind Med 1996;30:362- 8.

14. Jabelecki C, Andary M, So Y, Wilkins D, Williams F. AAEM Quality Assurance Committee: Literature review of the usefulness of nerve conduction studies and electromyography for the evaluation of patients with carpal tunnel syndrome. Muscle Nerve 1993;16:1392-1414.

15. D'Arcy CA, McGee S. Does this patient have carpal tunnel syndrome? JAMA 2000;283: 3110-17.

16. Bland JDP. Carpal tunnel syndrome: clinical review. BMJ 2007;335:343-6.

17. Rempel D, Evanoff B, Amadio PC, et al. Consensus criteria for the classification of carpal tunnel syndrome in epidemiologic studies. Am J Public Health 1998;88:1447-51.

18. G Ghugare, P Das, M Chitle, R Singh. Peroneal nerve palsy and its electrophysiological diagnosis. Published by Dept of Phyiology, MGIMS, Sevagram, Wardah- 442120.
19. Landau ME, Campbell WW. Clinical feature and electrodiagnosis of ulner neuropathies. Phys Med Rehabil Clin N Am 24 (2013) 49-66.

20. Preston DC, Shapiro BE: Peroneal Nerve Palsy. In: Preston DC, Shapiro BE eds, Electromyography and neuromuscular disorders : ClinicalElectrophysiologic Correlations 2nd ed, Philadelphia, Elsevier 2005:343-354.

21. Misra UK, Kalita J: Sacral plexus and its terminal nerve branches. In: Misra UK, Kalita $\mathrm{J}$ eds, Clinical Neurophysiology 2nd ed, New Delhi, Elsevier 2005:78-87.

22. Islam $Z$, Jacobs BC, Islam MB, Mohammad QD, Diorditsa S, Endtz HP. High incidence of Guillain-Barre syndrome in children, Bangladesh. Emerg Infect Dis 2011;17:1317-8.

23. Islam $Z$, Jacobs BC, van Belkum A, Mohammad $\mathrm{QD}$, Islam MB, Herbrink $\mathrm{P}$ et al. Axonal variant of Guillain-Barre syndrome associated with Campylobacter infection in Bangladesh. Neurology 2010;74:581-7.

24. Nadir ZK, Narullah M (1998) Electrodiagnostic study of 40 cases presenting as Guillain Barre Syndrome. Pak J Neurol 4: 50-54.

25. Yuki N, Hartung HP. Guillain Barre Syndrome. N Engl J Med 2012, 363 : 2294-304.

26. Dyck PJ, Prineas J, Pollard JD. Chronic inflammatory demyelinating polyradiculoneuropathy. In: Dyck PJ, Thomas PK, Griffin JW, et al, eds. Peripheral neuropathy. 3rd ed. Philadelphia: WB Saunders, 1993:1498-517.

27. Dyck PJ, Litchy WJ, Kratz KM, et al. A plasma exchange versus immune globulin infusion trial in chronic inflammatory demyelinating polyradiculoneuropathy. Ann Neurol 1994; 36: $838-45$.

28. Clare WA, Pamela JS. Motor neuron disease: a practical update on diagnosis and management. Clinical Medicine 2010, 10 (3): 252-58.

29. Stallberg E. Clinical electrophysiology in myasthenia gravis. Journal of Neurology, Neurosurgery, and Psychiatry, 1980, 43, 622-633. 